\title{
Analysis of acoustic resonator with shape deformation using finite element method
}

\author{
G M KALMSE ${ }^{\mathrm{a}}$, AJAY CHAUDHARI ${ }^{\mathrm{b}}$ and P B PATIL ${ }^{\mathrm{b}}$ \\ ${ }^{\text {a }}$ Science College, PB No. 62, Nanded 431603, India \\ ${ }^{\mathrm{b}}$ Department of Physics, Dr B A M University, Aurangabad 431 004, India \\ e-mail: bamuaur@bom4.vsnl.net.in
}

MS received 23 September 1999

\begin{abstract}
An acoustic resonator with shape deformation has been analysed using the finite element method. The shape deformation is such that the volume of the resonator remains constant. The effect of deformation on the resonant frequencies is studied. Deformation splits the degenerate frequencies.
\end{abstract}

Keywords. Finite element method; acoustic resonator; degenerate frequency.

\section{Introduction}

Acoustic resonators are mainly used to obtain information regarding the reflection and transmission coefficients of acoustic filters. We can use them to analyse the resonance in fuel tanks and musical instruments. Resonators are also used to determine the absorption coefficient of sound in different gases and liquids. Different geometrical structures have different resonating properties. Hence the need to analyse any possible new structure exists all the time. Extensive calculations have been made for cylindrical and spherical resonators to determine the resonant frequency and pressure in the immediate vicinity of the aperture. These include the work by Bjerke \& Van Bladel (1964), Van Bladel (1964, 1969), Edmonds (1961), and Ingard (1953). Microstrip resonators with inclined walls and bends have been analysed by Karkare et al (1996) and Chaudhari \& Patil (1997). However, nobody has reported the splitting of degenerate frequencies. The aim of this paper is to study the effect of deformation on degenerate and non-degenerate frequencies.

In this paper, the effect of shape deformation on a cubical resonator is investigated. Shape deformation is considered with increase in the sides of one face so that edges which were perpendicular to the face before deformation become oblique. Resonance frequencies are calculated for different extents of deformation and this variation is tabulated.

\section{Statement of the problem}

Consider a three-dimensional resonator in the form of a cube with acoustically stiff walls and with the geometrical dimensions $a, b$ and $c$, and with coordinate axes parallel to the 


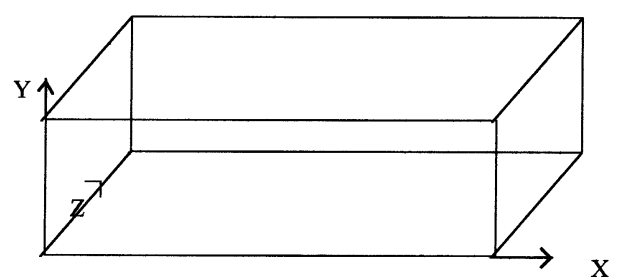

(a)

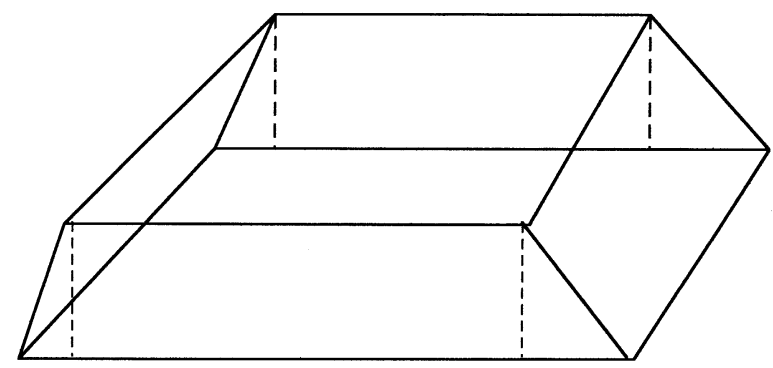

(b)
Figure 1. Undeformed (a) and shape deformed (b) acoustic resonators.

edges as shown in figure 1a. The boundaries are described by

$$
\begin{array}{ll}
x=0, & x=a, \\
y=0, & y=b, \\
z=0, & z=c .
\end{array}
$$

Let the resonator be deformed so that the shape is changed by extending its bottom surface on both sides as shown in figure $1 \mathrm{~b}$. Now, some of the boundary surfaces are not parallel to the coordinate planes.

If $V$ is the volume bounded by the rigid walls then the pressure satisfies the equation (Rayleigh 1945; Bjerke \& Van Bladel 1964; Van Bladel 1964, 1969)

$$
\nabla^{2} P+K^{2} P=0, \quad \text { over volume } \Omega,
$$

with boundary conditions

$$
\partial P / \partial n=0, \quad \text { over surface } \Gamma,
$$

where $\partial P / \partial n$ denotes normal derivative operator.

\section{Variational formulation}

To develop the functional $\Pi$ for the variational formulation of the problem described by (1) with boundary condition (2), multiply (1) by a scalar weight function $V$ and integrate it over the domain $\Omega$ of the resonator (Reddy 1986; Akin 1988).

$$
\Pi=\int_{\Omega} V\left(\nabla^{2} P\right) \mathrm{d} \Omega+K^{2} \int_{\Omega} V P \mathrm{~d} \Omega .
$$


By using Green's identity,

$$
\int_{\Omega} G\left(\nabla^{2} F\right) \mathrm{d} \Omega=-\int_{\Omega}(\nabla G \cdot \nabla F) \mathrm{d} \Omega+\int_{\Gamma} \frac{\partial F}{\partial n} G \mathrm{~d} \Gamma,
$$

and

$$
\frac{\partial}{\partial n}=n \cdot \nabla=n_{x} \frac{\partial}{\partial x}+n_{y} \frac{\partial}{\partial y}+n_{z} \frac{\partial}{\partial z} .
$$

Equation (3) reduces to

$$
\Pi=-\int_{\Omega}(\nabla V . \nabla P) \mathrm{d} \Omega+K^{2} \int_{\Omega} V P \mathrm{~d} \Omega+\int_{\Gamma} V(\nabla P \cdot n) \mathrm{d} \Gamma .
$$

The boundary term in (6) vanishes due to the boundary condition given by (2). Thus

$$
\Pi=-\int_{\Omega}(\nabla V \cdot \nabla P) \mathrm{d} \Omega+K^{2} \int_{\Omega} V P \mathrm{~d} \Omega .
$$

Substituting $P=V$ and dividing the terms involving $V$ and $P$ by two, we get the function for (1),

$$
\Pi=\frac{1}{2}\left[\int_{\Omega}(\nabla P \cdot \nabla P) \mathrm{d} \Omega-K^{2} \int_{\Omega} P P \mathrm{~d} \Omega\right]
$$

\section{Finite element formulation}

According to the finite element method, the domain of the resonator is divided into a number of volume elements. Here, tetrahedral elements with 20 nodes per element are used. The unknown $P$ at any point within an element is defined in terms of the nodal values $\left(P_{i}\right)$ using mapping function $F_{i}$ such that

$$
P=\sum_{i=1}^{N P} F_{i} P_{i}
$$

where NP is the number of nodes in an element, i.e. 20.

Equation (9) can be written in matrix form (Van Bladel 1964, 1969),

$$
\begin{aligned}
& P=\{F\}^{T}\left\{P^{e}\right\}=\left\{P^{e}\right\}^{T}\{F\}, \\
& \frac{\partial P}{\partial x}=\left\{\frac{\partial F}{\partial x}\right\}^{T}\left\{P^{e}\right\}=\left\{P^{e}\right\}^{T}\left\{\frac{\partial F}{\partial x}\right\},
\end{aligned}
$$

where

$$
\{F\}=\left[\begin{array}{c}
F_{1} \\
F_{2} \\
\vdots
\end{array}\right],
$$


and

$$
\left\{P^{e}\right\}=\left[\begin{array}{c}
P_{1} \\
P_{2} \\
\vdots
\end{array}\right] .
$$

The functional for the element can be written as

$$
\Pi^{e}=\frac{1}{2}\left[\int_{\Omega^{e}}(\nabla P \cdot \nabla P) \mathrm{d} \Omega-K^{2} \int_{\Omega^{e}} P P \mathrm{~d} \Omega\right] .
$$

Using (9) and (11), (14) becomes

$$
\begin{aligned}
\Pi^{e}= & \int_{\Omega^{e}} \frac{1}{2}\left[\left\{P^{e}\right\}^{T}\left\{\frac{\partial F}{\partial x}\right\} \cdot\left\{\frac{\partial F}{\partial x}\right\}^{T}\left\{P^{e}\right\}+\left\{P^{e}\right\}^{T}\left\{\frac{\partial F}{\partial y}\right\} \cdot\left\{\frac{\partial F}{\partial y}\right\}^{T}\left\{P^{e}\right\}\right. \\
& \left.+\left\{P^{e}\right\}^{T}\left\{\frac{\partial F}{\partial z}\right\} \cdot\left\{\frac{\partial F}{\partial z}\right\}^{T}\left\{P^{e}\right\}\right] \mathrm{d} x \mathrm{~d} y \mathrm{~d} z \\
& -K^{2} \int_{\Omega^{e}} \frac{1}{2}\left[\left\{P^{e}\right\}^{T}\{F\} \cdot\{F\}^{T}\left\{P^{e}\right\}\right] \mathrm{d} x \mathrm{~d} y \mathrm{~d} z .
\end{aligned}
$$

Taking $\left\{P^{e}\right\}^{T}$ as a common premultiplier and $\left\{P^{e}\right\}$ as a common postmultiplier,

$$
\begin{aligned}
\Pi^{e}=\frac{1}{2}\left\{P^{e}\right\}^{T}\left\{\int _ { \Omega ^ { e } } \left[\left\{\frac{\partial F}{\partial x}\right\} \cdot\right.\right. & \left\{\frac{\partial F}{\partial x}\right\}^{T}+\left\{\frac{\partial F}{\partial y}\right\} \cdot\left\{\frac{\partial F}{\partial y}\right\}^{T}+\left\{\frac{\partial F}{\partial z}\right\} \cdot\left\{\frac{\partial F}{\partial z}\right\}^{T} \\
& \left.\left.-K^{2}\{F\} \cdot\{F\}^{T}\right] \mathrm{~d} x \mathrm{~d} y \mathrm{~d} z\right\}\left\{P^{e}\right\} .
\end{aligned}
$$

In compact notation form,

$$
\Pi^{e}=\left\{P^{e}\right\}^{T}\left[S^{e}\right]\left\{P^{e}\right\}-K^{2}\left\{P^{e}\right\}^{T}\left[T^{e}\right]\left\{P^{e}\right\}
$$

where

$$
\left[S^{e}\right]=\int_{\Omega^{e}}\left[\left\{\frac{\partial F}{\partial x}\right\} \cdot\left\{\frac{\partial F}{\partial x}\right\}^{T}+\left\{\frac{\partial F}{\partial y}\right\} \cdot\left\{\frac{\partial F}{\partial y}\right\}^{T}+\left\{\frac{\partial F}{\partial z}\right\} \cdot\left\{\frac{\partial F}{\partial z}\right\}^{T}\right]
$$

and

$$
\left[T^{e}\right]=\int_{\Omega^{e}}\left[\{F\} \cdot\{F\}^{T}\right] \mathrm{d} x \mathrm{~d} y \mathrm{~d} z .
$$

The functional $\Pi$ for the whole region $\Omega$ is given by,

$$
\Pi=\frac{1}{2} \sum\{P\}^{T}[S]\{P\}-K^{2}\{P\}^{T}[T]\{P\},
$$

where $\{P\}$ is the matrix of the nodal values of the entire system and $[S]$ and $[T]$ are obtained from assembling the element matrices $\left[S^{e}\right]$ and $\left[T^{e}\right]$ respectively. Variation of (18) with respect to nodal values leads to an eigenvalue equation. Minimizing of $\Pi$ i.e. $\partial \Pi / \partial\{P\}=0$ 
Table 1. Square of the propagation constant $\left(K^{2}\right)$ for a shape deformed acoustic resonator.

\begin{tabular}{lccccc}
\hline & \multicolumn{5}{c}{ Height of resonator reduced by units } \\
\cline { 2 - 6 } Undeformed resonator & \multicolumn{1}{c}{0.1} & 0.2 & 0.3 & 0.4 & \multicolumn{1}{c}{0.5} \\
\hline 9.94 & 7.96 & 6.15 & 4.59 & 3.28 & 2.19 \\
9.94 & 9.94 & 9.94 & 9.94 & 9.94 & 7.20 \\
9.94 & 12.23 & 14.96 & 14.57 & 11.30 & 9.94 \\
19.95 & 17.96 & 16.14 & 15.37 & 13.25 & 12.15 \\
19.95 & 20.65 & 22.42 & 23.82 & 20.69 & 14.04 \\
19.95 & 22.25 & 24.99 & 25.36 & 21.27 & 17.18 \\
30.03 & 30.74 & 28.42 & 25.48 & 31.29 & 25.41 \\
48.00 & 37.32 & 32.55 & 34.15 & 31.63 & 28.78 \\
48.00 & 47.27 & 38.38 & 35.51 & 41.38 & 40.16 \\
48.00 & 47.99 & 48.07 & 48.00 & 41.67 & 43.42 \\
\hline
\end{tabular}

gives the following eigenvalue equation

$$
[S]\{P\}-K^{2}[T]\{P\}=0 .
$$

By solving this eigenvalue equation using subspace iteration method (Bathe \& Wilson 1987), the eigenvalues i.e. $\mathbf{K}^{2}$ and eigenvectors, i.e. nodal values can be obtained.

\section{Numerical calculations}

Consider a cubical resonator with each side equal to unity. It is divided into 48 hexahedral elements with 20 nodes. Quadratic mapping functions are used. This is an undeformed resonator and the eigenvalues and eigenvectors are obtained.

The resonator is now deformed in such a way that its height is decreased without change in the volume of the resonator. To maintain the volume constant, the sides of the bottom face are suitably increased. Decrease in height is considered as $0.1,0.2,0.3,0.4$ and 0.5 units. For every decrease in height, the eigenvalues and eigenvectors are obtained. The eigenvalues for an undeformed resonator and for different decreases in height are given in table 1.

From the eigenvalues of the undeformed resonator, it is observed that some frequencies are degenerate, for example, 9.94, 19.95, 48 etc. The deformation has raised the degeneracy and split the degenerate state into three different frequencies. For the lowest $K^{2}$, it is seen that, with decrease in height, one $K^{2}$ value almost remains constant up to 0.4 units decrease in height, while another $K^{2}$ value decreases continuously with decrease of height and a third increases and then decreases.

It is also clear that deformation lowers the fundamental frequency. At 0.3 and 0.4 units, the frequencies of different modes move closer. For the third degenerate frequency, the deformation gives rise to double degeneracy (approximately) at different frequencies for different extents of deformation. For 0.1 units it is about 48, while for 0.4 units it is about 41.5 .

\section{Conclusion}

Shape deformation in a cubical acoustic resonator splits the degenerate frequencies. 


\section{References}

Akin J E 1988 Application and implementation of finite element method (New York: Academic Press)

Bathe K J, Wilson E 1987 Numerical methods in finite element analysis (New Delhi: Prentice Hall of India)

Bjerke C, Van Bladel J 1964 Excitation of a circular cylindrical cavity by a circular waveguide. $J$. Acoust. Soc. Am. 36: 1095-1099

Chaudhari A S, Patil P B 1997 Analysis of deformed microstrip resonator using finite element method. Sädhanā 22: 649-657

Edmonds P D 1961 On correlating the loss factors of cylindrical and spherical resonators. J. Acoust. Soc. Am. 33: 615-622

Ingard U 1953a On the theory and design of acoustic resonators. J. Acoust. Soc. Am. 25: 1037-1062

Ingard U 1953b Near field of a Helmholtz resonator exposed to plane wave. J. Acoust. Soc. Am. 25: 1062-1067

Karkare M M, Chaudhari A S, Patil P B 1996 Analysis of bent microstrip resonator using finite element method. Indian J. Phys. B70: 487-494

Rayleigh J W S 1945 The theory of sound (II) (New York: Dover)

Reddy J N 1986 An introduction to finite element method (New York: McGraw Hill)

Van Bladel J 1964 Excitation of resonant cavity by ducts of small cross-sectional dimensions. $J$. Acoust. Soc. Am. 36: 1090-1094

Van Bladel J 1969 Coupling through small apertures with an application to Helmholtz resonators. J. Acoust. Soc. Am. 45: 604-613 